\title{
Validação inicial do índice de necessidade de atenção à saúde bucal para as equipes de saúde bucal na estratégia de saúde da família
}

\author{
Initial validation of the index of oral healtcare needs \\ for oral health teams in the family healthcare strategy
}

Leonardo Carnut ${ }^{1}$

Leonardo Vilar Filgueiras ${ }^{2}$

Nilcema Figueiredo ${ }^{3}$

Paulo Sávio Angeiras de Goes ${ }^{1}$

\footnotetext{
${ }^{1}$ Faculdade de Odontologia, Universidade de Pernambuco. Avenida General Newton Cavalcanti, Tabatinga. 54753-901 Camaragibe PE. leonardo.carnut@gmail.com ${ }^{2}$ Departamento de Atenção à Saúde, Secretaria de Saúde de Camaragibe, Prefeitura Municipal de Camaragibe. ${ }^{3}$ Diretoria Geral de Assistência à Saúde, Secretaria de Saúde, Prefeitura da Cidade do Recife.
}

\begin{abstract}
This survey set out to validate the Index of Oral Healthcare Needs (IOHN), based on a pre-defined algorithm of the social status of families. The validation process was divided into two phases, namely a face validation and a construct validation. In the latter, data on caries experience, toothache and access to oral health services was collected. To validate the index a random, stratified sample of 412 children aged 3-5 and 712 was obtained, based on the IOHCN algorithm, all the children being from the areas of Recife covered by the family healthcare program. The analysis consisted of a descriptive and an analytical phase, adopting a 5\% level of significance. The index was considered by an expert committee to have good face validity. The convergent construct validation was associated with a decay component of dmft ( $p=0.03)$ and DMFT ( $p=0.01)$; the divergent construct validation was associated with access to oral care $(p=0.001)$ and filled component of dmft ( $p=0.05)$, showing no association with the filled component of DMFT. The Index of Oral Healthcare Needs was shown to have good initial validation and canbe used as a useful tool in the planning of dental care at a local level. Key words Epidemiologic surveillance, Equity in health, Public health dentistry
\end{abstract}

Resumo Este trabalho objetivou validar o Índice de Necessidade de Atenção à Saúde Bucal (INASB), a partir de um algoritmo pré-definido baseado nas condições sociais das famílias. Utilizaram-se informações obtidas na ficha $A$ do sistema de informação da atenção básica. A validação do indice foi realizada através da validação de face e de construto, e nesta foram coletados dados de experiência de cárie, dor de dente e acesso aos serviços de saúde bucal através de uma amostra aleatória, estratificada e baseada no indice de 412 crianças nas faixas etárias 3-5 e 7-12 anos em Recife-PE. Para análise foi utilizado o nível de significância de 5\%. O indice foi considerado adequado na validação de face. $\mathrm{Na}$ validação de construto convergente foi associado ao componente cariado do ceo-d $(p=0,03) e$ CPO-D ( $p=0,01)$; e na divergente foi associado ao acesso ao dentista $(p=0,001)$ e ao componente obturado do ceo-d ( $p=0,05)$ não sendo associado ao componente obturado do CPO-D. O Índice de Necessidade de Atenção à Saúde Bucal demonstrou possuir uma boa validade inicial podendo ser usado como instrumento de programação para as equipes de saúde bucal da família.

Palavras-chave Vigilância epidemiológica, Equidade em saúde, Odontologia em saúde pública 


\section{Introdução}

Desde sua inserção até os dias atuais, as ações de odontologia vêm se consolidando no SUS ${ }^{1}$, no entanto, a equidade no acesso as suas ações ainda é um problema a ser enfrentado, principalmente devido à íntima relação das doenças bucais e condições socioeconômicas desfavoráveis ${ }^{2-7}$. Vários autores $^{2-7}$ descreveram as evidências da relação entre cárie dentária (e suas conseqüências) com os determinantes mais distais da vida coletiva. Entre as condições estudadas, algumas têm sido consideradas mais fortes na determinação da cárie dentária como a baixa escolaridade dos pais, em especial a da mãe, e a baixa renda familiar, ${ }^{3,4}$.

O SUS legalmente preconiza o uso da equidade como forma de dirimir as iniquidades provocadas por estas condições sociais adversas ${ }^{8} . \mathrm{Na}$ práxis do acesso aos serviços de saúde, o uso deste princípio tende a ser uma alternativa factível do ponto de vista local considerando que as desigualdades sociais refletem quase sempre no padrão de saúde-doença da população ${ }^{9}$. Mais especificamente em relação ao acesso, a lei do cuidado inverso em saúde tende a prevalecer em sociedades iníquas favorecendo o acesso aos serviços de saúde àqueles que detêm melhor condição social ${ }^{10}$. Logo, pautar o processo de trabalho em saúde praticando equidade é fundamental, e para isso é necessário o uso de informações sobre as condições de vida da população. Estas informações devem servir de base para análise de situação de saúde-doença de cada coletividade, como também para programar as ações visando quem mais precisa ${ }^{11}$. Resumindo: deve-se usar a informação para a ação ${ }^{12}$ (garantindo que as ações desenvolvidas sejam equânimes).

Uma forma simples de captar informações em saúde no SUS pode ser realizada através dos Sistemas de Informação em Saúde (SIS), alguns sistemas, como por exemplo o SIA-SUS, registram dados de saúde bucal. Entretanto, estas informações são basicamente procedimentais e refletem uma lógica administrativa, servindo muitas vezes para caracterizar práticas assistenciais, ou no máximo, formular indicadores de proces$\mathrm{so}^{13}$, os quais teriam uso limitado no planejamento das ações. Para a obtenção de diagnósticos populacionais de saúde bucal em unidades de saúde, ou até mesmo para a construção de indicadores de resultados, se faz imprescindível a informação epidemiológica, que atualmente, não está disponível nos SIS para o nível local ${ }^{13}$. Mesmo com esforços para inclusão de dados epidemiológicos bucais no SIAB (através da Ficha DSaúde Bucal), o registro das informações em saú- de bucal na atenção básica ainda se restringe àquelas de caráter administrativo-procedimental ${ }^{14}$.

Portanto, há o desafio de se obter informações epidemiológicas em saúde bucal no nível local. Esta informação é um insumo essencial ao planejamento e programação das ações. Para Mota e Carvalho ${ }^{11}$ a informação epidemiológica, quando fornecida com precisão ${ }^{15}$, é de importância capital para a institucionalização de um modelo de vigilância à saúde como se deseja no SUS, pois tende a revelar perfis e tendências nas condições de saúde, sendo assim aplicável às atividades de vigilância ${ }^{11}$. Partindo-se desse pressuposto, seria de se esperar que o cirurgião-dentista, que atue na atenção básica, dispusesse dos conhecimentos necessários para realizar levantamentos epidemiológicos locais, todavia a filosofia de formação desse profissional reforça a prática assistencialista ${ }^{16}$ ocasionando dois grandes entraves na institucionalização do modelo de vigilância: a incipiência do trabalho com informações em saúde, e por consequência, a ausência de planejamento e programação local ${ }^{13,17,18}$.

Levando-se em consideração esses problemas, alguns autores ${ }^{19-21}$ sugeriram índices de necessidade de tratamento odontológico que orientam o processo de atenção, auxiliando os cirurgiõesdentistas na programação de suas demandas. Embora estes índices signifiquem um avanço, os mesmos refletem uma ótica biomédica valorizando a doença em detrimento do conceito ampliado de saúde, utilizando para isto apenas o diagnóstico clínico normativo. Com base nessas questões o presente estudo objetivou a validação inicial do Îndice de Necessidade de Atenção à Saúde Bucal (INASB) tomando como hipótese que as informações sociais disponíveis na ficha-A do SIAB poderiam estimar a informação epidemiológica (em termos de cárie dentária - problema prioritário da saúde bucal) em uma população adstrita a uma equipe de saúde bucal da estratégia de saúde da família.

\section{Metodologia}

Esse estudo foi desenvolvido na Cidade do Recife-PE entre abril e agosto de 2008 em uma Unidade de Saúde da Família (USF) com Equipe de Saúde Bucal (ESB) na proporção de 2:1, sendo a ESB modalidade II. A referida USF foi escolhida por se constituir em uma referência para atividades de preceptoria, graduação e pós-graduação na área.

O INASB foi um índice desenvolvido com o intuito de classificar as famílias de áreas adstritas à estratégia de saúde da família baseando-se nas 
informações sociais encontradas na ficha-A do SIAB (tipo de moradia e escolaridade materna). Estas variáveis foram selecionadas pela facilidade de serem obtidas e estarem associadas às condições de saúde bucal ${ }^{2-7}$. Para a construção do INASB foi hipotetizado um algoritmo (Quadro 1) que classifica como "alto risco" sempre que a condição de maior vulnerabilidade social estiver presente. Por exemplo: se a mãe é analfabeta ou mora em casa de material reciclado (portanto uma condição de alto risco) leva a esta família ser classificada como alto risco mesmo na presença de outra condição menos vulnerável.

A validação de medidas para serem utilizadas em estudos epidemiológicos está associada à validade interna desses estudos. Para o presente estudo foi seguido o protocolo de validação proposto por Lucena et $\mathrm{al}^{22}$ onde é preconizado os estágios de validação de face, de conteúdo e de construto, bem como a reprodutibilidade da medida.

Para a validação de face o algoritmo foi submetido a um grupo de cinco especialistas em odontologia e saúde coletiva, os quais concensuaram com a construção proposta de acordo com a evidência teórica proposta, formulada para o índice. Por não se tratar de questões ou quesitos para aplicação a um interlocutor, a validação de conteúdo não se aplicaria ao caso do índice proposto (não sendo necessário medidas de consistência interna como $\alpha$-cronbach). Para a validação de construto convergente foi testado se ní-

Quadro 1. Variáveis analisadas: definição e operacionalização.

\begin{tabular}{|c|c|c|c|}
\hline Variáveis & Definição & Tipo de dado & Operacionalização \\
\hline \multirow[t]{5}{*}{$\begin{array}{l}\text { Índice de Necessidade } \\
\text { Atenção à Saúde Bucal } \\
\text { (INASB) }\end{array}$} & $\begin{array}{l}\text { Obtido pelo algoritmo: } \\
\text { INASB = tipo de moradia (TM) } \\
+ \text { escolaridade materna }(\mathrm{EM})\end{array}$ & \multirow[t]{5}{*}{ Não-clínico } & \multirow{5}{*}{$\begin{array}{l}\text { Tipo de moradia* (TM) } \\
\text { alto risco } \\
5 \text { - material reciclado } \\
4 \text { - madeira } \\
3 \text { - taipa não-revestida } \\
2 \text { - taipa revestida } \\
\text { médio risco } \\
3 \text { - taipa não-revestida } \\
2 \text { - taipa revestida } \\
\text { baixo risco } \\
1 \text { - tijolo/adobe } \\
\text { Educação materna* } \\
\text { alto risco } \\
0 \text { - analfabeta } \\
1 \text { - alfabetizada } \\
\text { médio risco } \\
2 \text { - } 1^{\text {a }} \text { a a } 4^{\text {a }} \text {. Série } \\
\text { baixo risco } \\
3 \text { - } 5^{\text {a }} \text { a } 8^{\text {a }} \text {. Série } \\
4-2^{\circ} \text {. grau em diante }\end{array}$} \\
\hline & $\mathrm{TM}$ & & \\
\hline & $\begin{array}{cll}\text { INASB alto } & \text { alto risco } & \text { alto risco } \\
& \text { alto risco } & \text { médio risco } \\
\text { alto risco } & \text { baixo risco } \\
& \text { médio risco } & \text { alto risco } \\
& \text { baixo risco } & \text { alto risco } \\
\text { INASB médio } & \text { médio risco } & \text { médio risco }\end{array}$ & & \\
\hline & $\begin{array}{lll} & \begin{array}{l}\text { médio risco } \\
\text { baixo risco }\end{array} & \text { baixo risco } \\
\text { médio risco }\end{array}$ & & \\
\hline & & & \\
\hline Sexo & $\begin{array}{l}\text { Distinção entre os seres vivos em relação } \\
\text { à função reprodutora }\end{array}$ & Não-clínico & $\begin{array}{l}1-\text { Masculino } \\
2-\text { Feminino }\end{array}$ \\
\hline Idade & $\begin{array}{l}\text { Tempo de vida desde o nascimento até o } \\
\text { determinado momento }\end{array}$ & Não-clínico & Número absoluto (em anos) \\
\hline $\begin{array}{l}\text { Acesso ao dentista nos } \\
\text { últimos } 12 \text { meses }\end{array}$ & $\begin{array}{l}\text { Se o indivíduo já foi a uma consulta } \\
\text { odontológica nos últimos } 12 \text { meses }\end{array}$ & Não-clínico & $\begin{array}{l}1-\operatorname{Sim} \\
2-\mathrm{Não}\end{array}$ \\
\hline $\begin{array}{l}\text { Dor de dente nos } \\
\text { últimos } 6 \text { meses }\end{array}$ & \multirow{2}{*}{$\begin{array}{l}\text { Se o indivíduo sentiu alguma dor de dentes } \\
\text { nos últimos seis meses } \\
\text { Índice de dentes cariados, com extração } \\
\text { indicada e obturados para dentes decíduos }\end{array}$} & Não-clínico & $\begin{array}{l}1-\text { Sim } \\
2-\text { Não }\end{array}$ \\
\hline ceo-d & & Clínico & Número absoluto \\
\hline CPO-D & $\begin{array}{l}\text { Índice de dentes cariados, perdidos e } \\
\text { obturados para a dentição permanente }\end{array}$ & Clínico & Número absoluto \\
\hline
\end{tabular}


veis maiores de cárie, medido pelo componente cariado do ceo-d e CPO-D e maior frequência de dor nos últimos seis meses estaria associado ou correlacionado com maior classificação na escala do INASB. Contrariamente, na validação de construto divergente foi testado se menor frequência de acesso nos últimos 12 meses e menores níveis de atenção odontológica medida pelo componente obturado do ceo-d e CPO-D reproduziam níveis mais elevados de risco na escala proposta pelo INASB.

A coleta de dados para o processo de validação se deu em duas fases metodologicamente distintas. A primeira fase constituiu-se na coleta de dados secundários a partir da ficha-A do Sistema de Informação da Atenção Básica (SIAB). Foi realizado um censo das famílias que possuíam indivíduos na idade de 5 e 12 anos (ao todo 130 famílias) cadastradas na USF, no entanto o número total de indivíduos nessas idades não foi o suficiente para retirar uma amostra e testar a associação. Para isso, resolveu-se ampliar as idades para duas faixas etárias (3-5 e 7-12 anos) que englobassem as idades-índices de 5 e 12 . Após recenseadas novamente em formulário padrão obteve-se 777 famílias com indivíduos nas faixas de 3-5 e 7-12 anos. Essas famílias foram classificadas segundo o algoritmo do INASB em 03 grupos distintos (alto risco, médio risco e baixo risco).

Uma vez as famílias recenseadas e separadas em 03 grupos (alto risco $=80$ famílias, médio risco = 153 famílias e baixo risco = 519 famílias) foram obtidas 03 amostras aleatórias, uma de cada um dos grupos com fins de testar a hipótese. Para tal, tomou-se como referência a prevalência de cárie dentária, para a região nordeste, na idade de 12 anos segundo dados do SBBrasil 2003 usando o programa Epi Info 6.04. Foi utilizada a fórmula para comparação de duas proporções com intervalo de confiança de $95 \%$ e erro de $5 \%$, obtendo-se uma amostra total de 438 indivíduos somando-se as faixas etárias de 3-5 e 712 anos estratificadas de acordo com o INASB. Os 438 indivíduos resultaram do sorteio de 518 famílias das 777 que tinham crianças nas faixas etárias alvo.

A segunda fase caracterizou-se pela coleta de dados primários epidemiológicos de cárie dentária na população de 3-5 e 7-12 anos sorteada. A unidade amostral primária constituiu-se nos domicílios. Para coletar os dados, três examinadores (cirurgiões-dentistas) e dois anotadores (01 auxiliar de saúde bucal e 01 técnico em saúde bucal) foram devidamente calibrados para este fim como descrito nos critérios de confiabilidade dos dados a seguir. A tomada do consentimento livre esclarecido do responsável foi conduzida primeiramente e após feita a coleta dos dados não-clínicos (condições sociais) e os clínicos (cárie dentária).

Para garantir a confiabilidade dos dados clínicos coletados, foi realizado o treinamento e a calibração dos examinadores, sendo a qualidade dos exames aferidos pelo Teste Kappa. Os resultados evidenciaram uma concordância (Kappainter $=0,87$ ) excelente seguindo os parâmetros estabelecidos por Cohen ${ }^{23}$. Em adição, o controle de qualidade dos dados foi realizado através do re-exame dos indivíduos no prazo entre dois dias (48 horas) até no máximo uma semana (01 semana) (calibração intra-examinador) cujo teste Kappa obtido alcançou o nível de excelência para os examinadores que participaram do estudo (Kappaintra1 $=0,87$, Kappaintra2 $=0,94$ e Kappaintra3 $=0,95)$. Observou-se ainda que a taxa de completude das fichas-A foi de $100 \%$, em adição, visando garantir a validade dos dados das condições sociais presentes na ficha-A, coletouse in loco os mesmos dados, que depois de submetidos à comparação com as informações contidas nestas fichas observou-se uma concordância considerada aceitáve ${ }^{23}($ Kappa $=0,50)$.

Dentre as variáveis analisadas, as mesmas foram divididas de acordo com a análise proposta pela metodologia do estudo em: variável dependente (INASB) e variáveis independentes (sexo, idade, dor de dente nos últimos 6 meses, acesso ao dentista nos últimos 12 meses e os componentes cariado e obturado dos ceo-d/CPOd). O Quadro 1 mostra esquematicamente a definição e a operacionalização das mesmas. A análise estatística dos dados coletados foi processada pelo Statistical Package for Social Science (SPSS) versão 11.0. A análise dos dados ocorreu em duas fases distintas: uma descritiva e outra analítica. $\mathrm{Na}$ fase descritiva foram feitas as distribuições de freqüências das variáveis e, quando apropriado, foram calculadas as medidas de tendência central, de dispersão e proporções. Na fase analítica/inferencial foram testadas as associações entre o INASB e as variáveis com distribuição não-normal utilizando-se o teste de KruskalWallis e Qui-quadrado. A avaliação da normalidade das variáveis continuas foi realizada utilizando o teste Kolmogorov-Smirnov (Teste KS). Para todas as análises foi considerado o nível de significância de 5\%.

Após análise do Comitê de Ética em Pesquisa da Universidade de Pernambuco a pesquisa foi aprovada como estando em consonância com a 
Ao final de três meses de coleta de dados primários foi obtida uma amostra de $412(\mathrm{n}=412)$ indivíduos entrevistados e examinados, totalizando um percentual de perda amostral de 5,9\% em relação à amostra calculada para o estudo.

Após a análise da amostra obtida, dentre as condições sociodemográficas, foi observado que, em relação ao sexo, 49,5\% dos indivíduos eram do sexo masculino (204) e 50,5\% do sexo feminino (208). Em relação à faixa etária pertencente, observou-se que $26,7 \%$ pertenciam à faixa etária de 3-5 anos de idade (110) e em contrapartida $73,3 \%$ dos indivíduos pertenciam à faixa etária de 7-12 anos (302). Para a classificação de risco a amostra se comportou como esperado pela estratificação com 15\% dos indivíduos pertencentes ao grupo de alto risco (62), 23,3\% pertencentes ao grupo de médio risco (96) e 61,7\% pertenciam ao grupo de baixo risco (254).

Em relação aos dados clínicos, obteve-se a média para o índice ceo-d de 1,86 e CPO-D de 0,63 dentes. A média do componente cariado para o ceo-d foi de $1,41(95 \%$ IC $=1,19-1,63, \mathrm{DP}=$ 2,28 ) enquanto que, para o CPO-D foi de 0,44 $(95 \% \mathrm{IC}=0,33-0,55, \mathrm{DP}=1,12)$. Quanto à composição percentual predominante tanto no ceo-d, quanto no CPO-D constituiu-se pelo componente cariado, atingindo $75,8 \%$ para dentes decíduos e $69,8 \%$ para dentes permanentes. Em seguida com maior proporção no montante do ceo-d/CPO-D segue o componente obturado com uma freqüência percentual de $12,3 \%$ para o ceo-d e $25,3 \%$ para o CPO-D. Em terceiro, segue o componente perdido com $11,2 \%$ para o índice ceo-d e 4,7\% para o CPO-D.

Em uma análise exploratória com o Teste KS, verificou-se a normalidade dos dados e observou-se que os mesmos se distribuíam de forma não-normal. Na validação de construto convergente pelo teste de Kruskal-Wallis, (tomando-se como variável dependente INASB) observou-se uma relação estatisticamente significante tanto com o componente cariado do ceo-d $(\mathrm{p}=0,008)$ como no do CPO-D ( $p=0,013)$, no entanto para o componente obturado, a significância estatística apenas foi constatada no ceo-d $(\mathrm{p}=0,044)$ (Tabelas 1 e 2). Observou-se que a freqüência

dos componentes cariado e obturado do ceo-d obtiveram associação estatisticamente significante com a classificação INASB, enquanto que para o CPO-D apenas o componente cariado demonstrou significância. O Acesso ao Dentista nos últimos 12 meses demonstrou forte associação estatística com a classificação proposta pelo índice, como pode ser observado na Tabela 3.

\section{Discussão}

O presente estudo demonstrou uma associação entre a classificação de risco social atribuído às famílias através do INASB, obtido com informações da ficha-A, com a cárie dentária, podendo se caracterizar como uma medida que auxilie as ESB da ESF a programar ações para a população infantil de áreas cobertas pela estratégia. A proposição deste índice foi baseado na sólida associação demonstrada por vários estudos entre condições sociais e saúde bucal (entre a qual está a cárie - problema prioritário de saúde bucal das populações) $)^{2-7}$.

A classificação de risco das famílias proposta pelo INASB foi associada ao componente cariado do ceo e CPO. Isto demonstra a validade ao

Tabela 1. Teste de Associação de Kruskal-Wallis para INASB em relação aos componentes cariado e obturado do ceo-d.

\begin{tabular}{lccccc}
\hline INASB & $\mathbf{N}$ & $\mathbf{c}$ & Valor de $\mathbf{p}$ & $\mathbf{o}$ & Valor de p \\
\hline Alto & 62 & 230,83 & $0,008^{*}$ & 189,7 & $0,044^{*}$ \\
Médio & 96 & 231,11 & & 201,0 & \\
Baixo & 254 & 193,70 & & 212,6 & \\
Total & $\mathbf{4 1 2}$ & - & & - & \\
\hline
\end{tabular}

Fonte: Pesquisa direta.

$\cdot(\mathrm{p}<0,05)$

Tabela 2. Teste de Associação de Kruskal-Wallis para INASB em relação aos componentes cariado e obturado do CPO-D.

\begin{tabular}{lccccc}
\hline INASB & $\mathbf{N}$ & \multicolumn{1}{c}{$\mathbf{c}$} & Valor de $\mathbf{p}$ & $\mathbf{o}$ & Valor de $\mathbf{p}$ \\
\hline Alto & 62 & 227,6 & $0,013^{*}$ & 200,0 & 0,539 \\
Médio & 96 & 216,9 & & 210,9 & \\
Baixo & 254 & 197,3 & & 206,3 & \\
Total & $\mathbf{4 1 2}$ & - & & - & \\
\hline
\end{tabular}

Fonte: Pesquisa direta. ${ }^{*}(\mathrm{p}<0,05)$ 
Tabela 3. Frequência dos componentes cariado e obturado (ceo-d/CPO-D), dor de dente e acesso do dentista nos últimos 12 meses em relação à classificação INASB.

\begin{tabular}{|c|c|c|c|c|c|c|c|c|c|c|}
\hline & \multicolumn{8}{|c|}{ Classificação INASB } & \multirow{3}{*}{$\chi^{2}$} & \multirow[b]{3}{*}{$\begin{array}{c}\text { Valor } \\
\text { de } p\end{array}$} \\
\hline & \multicolumn{2}{|c|}{ Baixo } & \multicolumn{2}{|c|}{ Médio } & \multicolumn{2}{|c|}{ Alto } & \multicolumn{2}{|c|}{ Total } & & \\
\hline & $\mathbf{N}$ & $\%$ & $\mathrm{~N}$ & $\%$ & $\mathbf{N}$ & $\%$ & $\mathbf{N}$ & $\%$ & & \\
\hline Frequência do componente cariado (ceo-d) & & & & & & & & & 6,77 & $0,03^{*}$ \\
\hline$c=0$ & 215 & 65,0 & 72 & 21,8 & 33 & 14,0 & 235 & 57,0 & & \\
\hline$c \geq 1$ & 39 & 48,1 & 24 & 29,6 & 29 & 16,4 & 177 & 43,0 & & \\
\hline Frequência do componente cariado (CPO-D) & & & & & & & & & 8,71 & $0,01^{*}$ \\
\hline $\mathrm{C}=0$ & 215 & 65,0 & 72 & 21,8 & 44 & 13,3 & 331 & 80,3 & & \\
\hline $\mathrm{C} \geq 1$ & 39 & 48,1 & 24 & 29,6 & 18 & 22,2 & 81 & 19,7 & & \\
\hline Frequência do componente obturado (ceo-d) & & & & & & & & & 5,96 & $0,05^{*}$ \\
\hline $\mathrm{o}=0$ & 214 & 59,6 & 86 & 24,0 & 59 & 16,4 & 359 & 87,1 & & \\
\hline $0 \geq 1$ & 40 & 54,8 & 10 & 28,8 & 3 & 16,4 & 53 & 12,9 & & \\
\hline Frequência do componente obturado (CPO-D) & & & & & & & & & 1,10 & 0,57 \\
\hline $\mathrm{O}=0$ & 230 & 61,7 & 85 & 22,8 & 58 & 15,5 & 373 & 90,5 & & \\
\hline $\mathrm{O} \geq 1$ & 24 & 61,5 & 11 & 28,2 & 4 & 10,3 & 39 & 9,5 & & \\
\hline Frequência de dor de dente & & & & & & & & & 2,57 & 0,27 \\
\hline $\operatorname{Sim}$ & 64 & 56,6 & 30 & 26,5 & 19 & 16,8 & 113 & 30,0 & & \\
\hline Não & 172 & 65,2 & 59 & 22,3 & 33 & 12,5 & 264 & 70,0 & & \\
\hline Acesso ao dentista nos últimos 12 meses & & & & & & & & & 18,39 & $0,001^{*}$ \\
\hline Sim & 125 & 69,1 & 40 & 22,1 & 16 & 8,8 & 181 & 43,9 & & \\
\hline Não & 111 & 56,1 & 52 & 26,3 & 35 & 17,7 & 198 & 48,1 & & \\
\hline Não lembro/Não Informado & 18 & 54,5 & 4 & 12,1 & 11 & 33,3 & 33 & 8,0 & & \\
\hline
\end{tabular}

Fonte: Pesquisa direta.

${ }^{*}(\mathrm{p}<0,05)$

índice, haja vista que há uma bem descrita associação entre condição sócio-econômica e cárie ${ }^{2-7}$. No entanto não foi evidenciado uma associação do índice e dor de dente, esta associação tem sido demonstrada ainda de forma contraditória na literatura, alguns estudos não tem evidenciado esta associação $0^{24}$, enquanto outros em população brasileira tem relatado a mesma ${ }^{3,6}$. Esta associação pode não ter sido evidenciada nesse estudo por duas razões: (1) devido aos níveis de ceo e $\mathrm{CPO}$ encontrados serem menores que média nacional para a região, demonstrando uma validação de construto convergente parcial; (2) bem como o fato de parte da amostra ser de indivíduos com dentição decídua, e serem raros os estudos que demonstram uma alta prevalência de dor de dente nesta dentição $0^{25}$.

Outro dado importante foi a fraca associação do componente obturado com o INASB. A instabilidade observada entre as associações do índice proposto e o componente obturado, a depender do teste utilizado, pode estar demonstrando que ao não programar sua demanda de forma equânime o serviço atinge a todos de forma igualitária, haja vista que a doença é maior nos indivíduos pertencentes às famílias de alto risco, produzindo desta forma desigualdades na assistência a saúde.

$\mathrm{O}$ fato descrito acima pode ser confirmado pela forte associação entre a classificação de risco proposta pelo INASB e a variável acesso ao Dentista nos últimos 12 meses. Evidenciou-se que entre os indivíduos pertencentes às famílias de alto risco, há quase o dobro de percentual de não ter acesso ao Dentista em relação àqueles pertencentes ao baixo risco. Embora a existência de uma discussão acadêmica em torno do conceito de acesso aos serviços de saúde, considerando as muitas dimensões que este pode ser avaliado ${ }^{26}$; o suplemento-saúde da PNAD/98 retratou o acesso/utilização de serviço sendo entendido como o encontro comportamental entre o usuário e o profissional de saúde que o conduz dentro do sistema. Evidenciou-se, na época, um grave problema odontológico nacional: 19\% dos brasileiros nunca tinham ido ao dentista, o que se analisado de acordo com a região, pode chegar ao número de 39 milhões de brasileiros ${ }^{27}$.

Mais recentemente, Rocha e Goes ${ }^{28}$ compararam o acesso de áreas cobertas e não cobertas por equipes de Saúde Bucal da ESF, no município de Campina Grande. Foi demonstrado um 
maior acesso para os indivíduos em áreas nãocobertas do que em áreas cobertas; revelando um importante papel dos fatores sociodemográficos, o que nos leva a refletir que embora a descentralização dos serviços tenha contribuído para o processo de superação das barreiras geográficas sobre o acesso aos serviços ${ }^{28}$, é fato que o acesso aos mesmos ainda são iníquos, devido à persistência causada pelas desigualdades sociais ${ }^{29}$.

Os achados deste estudo podem ser uma explicação para o fato de que as iniqüidades de acesso à saúde bucal ainda perdurem mesmo após o investimento maciço do governo federal na estruturação do Brasil Sorridente e o aumento do número das equipes de saúde bucal na estratégia de saúde da família. Ficando evidenciado, que a determinação equânime de quem necessita de atenção é falho; e demonstra uma falta de planejamento e programação das equipes de saúde bucal ao atender a população de sua própria área adstrita $^{17}$. Daí a contribuição que o presente índice pode trazer para as equipes de saúde bucal.

Ante o exposto, fica claro que o índice proposto pode representar uma boa ferramenta de programação para o nível local. Primeiro porque ele se fundamenta na implantação de ações baseadas no paradigma da eqüidade indo ao encontro do estabelecimento de diagnóstico de saúde, tomando a posição do individuo dentro do seu ambiente em contraste com os demais que são essencialmente baseados no modelo biomédiCO $^{19,20,21}$. Muito embora para sua validação inicial tenha se considerado os níveis de cárie medidos através de índices normativos como o ceo e CPO.

Além disso, as informações que subsidiam a formulação do presente índice podem ser retiradas da própria ficha-A que é preenchida nas visitas que o agente comunitário de saúde (ACS) faz às famílias de sua comunidade, devendo ser periodicamente atualizada ${ }^{30}$. Essas informações parecem servir para estimar cárie nas populações e não comprometem as atribuições legais do ACS, ressaltando-se que ao optar por exames de diagnóstico em saúde bucal, mesmo em se tratando de levantamentos epidemiológicos, isto é uma prerrogativa exclusiva do cirurgião-dentista como elencado em documentosoficiais ${ }^{31,32}$.

Mesmo com as soluções trazidas com a utilização do INASB deve-se salientar que o mesmo apresenta certas limitações. Validações mais robustas devem ser feitas em relação ao seu potencial de detecção das mudanças frente a interven- ções. As mudanças produzidas pelas intervenções devem ser avaliadas tanto na perspectiva quantitativa, bem como na perspectiva qualitativa. Nesta última o foco deve estar na compreensão das relações e das interpretações dos diversos atores sobre o processo propiciando uma análise do contexto onde essas intervenções acontecem. Desta forma a triangulação de métodos avaliativos, poderia ser usada devido à complexidade do objeto, e não pela linha epistemológica do observador ${ }^{33}$. Em adição, é importante que este trabalho seja replicado em outras faixas etárias e com outros agravos à saúde bucal para que seja avaliada sua estabilidade e coerência metodológica.

Contudo, vale observar as características que conferem validade ao presente estudo. O mesmo teve como base uma amostra calculada com critérios estatísticos coerentes, desde sua técnica de amostragem até a coleta de dados. Os critérios de exames são universalmente aceitos e o controle de qualidade dos dados foi atingido seguindo a metodologia de calibração proposta por diversos estudos e autores ${ }^{15,22,25}$ conferindo a confiabilidade necessária aos seus achados.

\section{Conclusões}

1. A Classificação de risco das famílias proposta pelo INASB esteve associada ao componente cariado do ceo e CPO o que demonstra que o índice ajuda na indicação das famílias que necessitam de maior atenção à saúde bucal, facilitando assim a programação da demanda com informações obtidas por instrumentos de uso cotidiano na atenção básica;

2. O acesso ao Dentista nos últimos 12 meses esteve associado à classificação proposta pelo índice, evidenciando-se desta maneira que crianças classificadas em "alto risco" tinham menor acesso ao Dentista quando comparadas com as de "baixo risco";

3. Foi demonstrado que o índice possui validade de construto convergente (componente c do ceo e CPO) e divergente para as variáveis analisadas (acesso aos serviços de saúde bucal, e componente o do ceo), o que demonstra a inversão do cuidado em saúde, observando-se que as crianças de famílias com melhores condições sociais (baixo risco) acessam mais os serviços odontológicos, contrariando assim a lógica da equidade. 


\section{Colaboradores}

Carnut L foi responsável pela coleta de dados, revista de literatura, confecção do artigo científico e concepção teórica do INASB em conjunto como os demais autores. Filgueiras LV trabalhou na coleta de dados e organização da revista de literatura. Figueiredo N co-orientou o trabalho, conduziu o processo de calibração dos examinadores e concepção teórica do INASB em conjunto como os demais autores. Goes PSA orientou o trabalho, delineou a metodologia adequada, corrigiu o artigo científico e trabalhou na concepção teórica do INASB em conjunto como os demais autores.

\section{Referências}

1. Araújo YP, Dimenstein M. Estrutura e organização do trabalho do cirurgião-dentista no PSF de municípios do Rio Grande do Norte. Cien Saude Colet 2006; 11(1):219-227.

2. Peres MA, Latorre MRDO, Sheiham A, Peres KG, Barros FC, Hernandez PG, Mass AMN, Romano AR, Victora CG. Determinantes sociais e biológicos da cárie dentária em crianças de 6 anos de idade: um estudo transversal aninhado numa coorte de nascidos vivos no Sul do Brasil. Rev Bras Epidemiol 2003; 6(4):293-306.

3. Bastos JLD, Nomura LH, Peres MA. Dental pain, socioeconomic status, and dental caries in young male adults from southern Brazil. Cad Saude Publica 2005; 21(5):1416-1423.

4. Meneghim MC, Kozlowski FC, Pereira AC, Ambrosano GMB, Meneghim ZMAP. Classificação socioeconômica e sua discussão em relação à prevalência de cárie e fluorose dentária. Cien Saude Colet 2007; 12(2):523-529.

5. Barabato PR, Nagano, HCN, Zanchet FN, Boing AF, Peres MA. Perdas dentárias e fatores sociais, demográficos e de serviços associados em adultos brasileiros: uma análise dos dados do Estudo Epidemiológico Nacional (Projeto SB Brasil 2002-2003). Cad Saude Publica 2007; 23(8):1803-1814.

6. Goes PSA, Watt RG, Hardy R, Sheiham A. The prevalence and severity of dental pain in 14-15 year old Brazilian schoolchildren. Community Dent Health 2007; 24(4):217-224

7. Gomes D, Da Ros MA. A etiologia da cárie no estilo de pensamento da ciência odontológica. Cien Saude Colet 2008; 13(3):1081-1090.

8. Brasil. Lei 8.080 de 19 de setembro 1990. Dispõe sobre as condições para a promoção, proteção e recuperação da saúde, a organização e o funcionamento dos serviços correspondentes e dá outras providências. Diário Oficial da União 1990; 12 set.

9. Barros MBA. Eqüidade e saúde. Epidemiol Serv Saude 2006; 15(4):5-6.

10. Hart JT. The Inverse Care Law. Lancet [serial on the Internet] $1971 \mathrm{Feb}$ [cited 2009 mar 11]:1(1):[about 10p.] Available from: C:|Users\Usuario\Downloads $\backslash$ The Lancet Saturday 27 February 1971.mht

11. Mota E, Carvalho DM. Sistemas de Informação em Saúde. In: Rouquayrol MZ. Epidemiologia e Saúde. Rio de Janeiro: MEDSI; 2003. p. 505-521.

12. Goes PSA. Vigilância à saúde bucal para o nível local - uma abordagem integrada para as equipes de saúde bucal da estratégia de saúde da família. In: Moysés ST, Kriger L; Moysés SJ. Saúde Bucal das Famílias. São Paulo: Artes Médicas; 2008. p. 258287. 
13. Barros SG, Chaves SCL. A utilização do sistema de informações ambulatoriais (SIA-SUS) como instrumento para caracterização das ações de saúde bucal. Epidemiol Serv Saude 2003; 12(1):41-51.

14. Ministério da Saúde. Secretaria de Atenção à Saúde. Departamento de Atenção Básica. Coordenação Nacional de Saúde Bucal. Proposta de Ficha DSaúde Bucal para o SIAB. Brasília: Ministério da Saúde; 2007. [documento para consulta pública].

15. Frias AC, Antunes JLF, Narvai PC. Precisão e validade de levantamentos epidemiológicos em saúde bucal: cárie dentária na Cidade de São Paulo, 2002. Rev bras epidemiol 2004; 7(2):144-154.

16. Rios ERG, Franchi KMB, Silva RM, Amorim RF, Costa NC. Senso comum, ciência e filosofia: elo dos saberes necessários à promoção da saúde. Cien Saude Colet 2007; 12(2):501-509.

17. Baldani MH, Fadel CB, Possamai T, Queiroz MGS. A inclusão da odontologia no Programa de Saúde da Família no Estado do Paraná. Cad Saude Publica 2005; 21(4):1026-1035.

18. Szwarcwald CL, Mendonça MHM, Andrade CLT. Indicadores de atenção básica em quatro municípios do Estado do Rio de Janeiro, 2005: resultados de inquérito domiciliar de base populacional. Cien Saude Colet 2006; 11(3):643-655.

19. Gushi LL, Rihs LB, Soares MC, Forni TIB, Vieira V, Wada RS, Sousa MLR. Cárie dentária e necessidades de tratamento em adolescentes do estado de São Paulo, 1998 e 2002. Rev Saude Publica 2008; 42(3):480-486.

20. Dumont AFS, Salla JT, Vilela MBL, Moraes PC, Lucas SD. Índice de necessidade de tratamento odontológico: o caso dos índios Xakriabá. Cien Saude Colet 2008; 13(3):1017-1022.

21. Vieira APGF, Saintrain MV. Validation of the Community Oral Health Index. Caries Res 2008; 42(4):239.

22. Lucena LBS, Kosminsky M, Costa LJ, Goes PSA. Validation of the Portuguese version of the RDC/ TMD Axis II questionnaire. Braz oral res 2006; 20(4):312-317.

23. Cohen J. A Coefficient of agreement for nominal scales. Educ Psychol Meas 1960; 20(3):37-46.

24. Bassols A, Bosch F, Campillo M, Canellas M, Banos JE. An epidemiological comparison of pain complaints in the general population of Catalonia (Spain). Pain 1999; 83(1):9-16.

25. Goes PSA,Watt RG, Hardy R, Sheiham A. Impacts of dental pain on daily activities of adolescents aged 14-15 years and their families. Acta Odontol Scand 2008; 66(1):7-12.

26. Penchansky R, Thomas W. The concept of access: definition and relationship to consumer satisfaction. Med Care 1981; 19(2):127-140.
27. Barros AJD, Bertoldi AD. Desigualdades na utilização e no acesso a serviços odontológicos: uma avaliação em nível nacional. Cien Saude Colet 2002; 7(4):709-717.

28. Rocha RACP, Goes PSA. Comparação do acesso aos Serviços de Saúde Bucal em áreas cobertas e não cobertas pela Estratégia Saúde da Família em Campina Grande - PB. Cad Saude Publica 2008; 24(12):2871-2880.

29. Roncalli AG. Epidemiologia e saúde bucal coletiva: um caminhar compartilhado. Cien Saude Colet 2006; 11(1):105-114.

30. Brasil. Ministério da Saúde. Secretaria de Atenção à Saúde. Departamento de Atenção Básica. SIAB Sistema de Informação da Atenção Básica. $4^{a}$ Reimp. Brasília: MS; 2003.

31. Brasil. Ministério da Saúde. Secretaria de Atenção à Saúde. Departamento de Atenção Básica. Coordenação Nacional de Saúde Bucal. Diretrizes da Política Nacional de Saúde Bucal. Brasília: MS; 2004.

32. Brasil. Ministério da Saúde. Secretaria de Atenção à Saúde. Departamento de Atenção Básica. Política Nacional de Atenção Básica. 4a ed. Brasília: MS; 2007.

33. Minayo MCS, Souza ER, Constantino P, Santos MC. Métodos Técnicas e Relações em Triangulação. In: Minayo MCS, Assis SG, Souza ER. Avaliação por Triangulação de Métodos - Abordagem de Programas Sociais. Rio de Janeiro: Editora Fiocruz; 2005. p. 71-103.

Artigo apresentado em 16/03/2009

Aprovado em 08/06/2009

Versão final apresentada em 02/07/2009 